\title{
Microstructure and Properties of Magnets Obtained by Hydrostatic Extrusion of $\mathrm{Nd}-\mathrm{Fe}-\mathrm{B}$ Powder
}

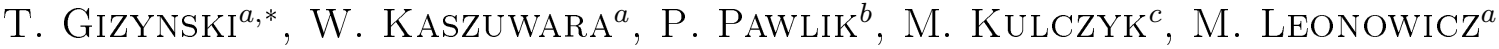 \\ AND B. MiChALSKI ${ }^{a}$ \\ ${ }^{a}$ Warsaw University of Technology, Faculty of Materials Science and Engineering, \\ Wołoska 141, 02-507 Warsaw, Poland \\ ${ }^{b}$ Częstochowa University of Technology, Institute of Physics, al. Armii Krajowej 19, 42-200 Częstochowa, Poland \\ ${ }^{c}$ Institute of High Pressure Physics, Polish Academy of Sciences, Sokołowska 29/37, 01-142 Warsaw, Poland
}

Nd-Fe-B MQ powder was initially densified in a copper capsule to reach about $60 \%$ of the theoretical density. Subsequently, three various processes of hydrostatic extrusion were conducted at room temperature. The values of true strain, obtained during the all three stages, were $1.38,0.89,0.94$, respectively. The investigation performed showed that the coercivity of the material decreases as the strain increases. Decrease of the remanence was observed only for the highest strains. Size of the particles was strongly reduced during the extrusion processes. X-ray diffraction did not show changes in the phase structure of the material. The Mössbauer study, of the sample extruded within all the three stages, showed existence of the $\mathrm{Nd}_{2} \mathrm{Fe}_{14} \mathrm{~B}$ phase and $16 \%$ of other phase. Analysis of magnetization versus temperature confirmed that the additional phase was ferromagnetic.

DOI: 10.12693/APhysPolA.127.626

PACS: $75.50 . \mathrm{Ww}$

\section{Introduction}

Hydrostatic extrusion (HE) is a modern, advanced method of plastic deformation [1]. This method is widely applied for fabrication ultra-fine-grained materials in a form of wires or rods. The results presented in [2] confirm that $\mathrm{HE}$ can also be used for densification of powders. The characteristic feature of $\mathrm{HE}$ is high speed of the process, which retards grain growth in a case of extrusion at high temperature. Among major advantages of $\mathrm{HE}$ process one can include triaxial state of stress acting at the material in the course of extrusion, which in a case of extrusion of bulk materials retards formation and propagation of cracks and in application for powders allows their good densification.

So far, only one group reported the application of $\mathrm{HE}$ for densification of $\mathrm{Nd}-\mathrm{Fe}-\mathrm{B}$ powders [3]. It was shown that generation of high deformation ratios, in the $\mathrm{HE}$ method, enables fabrication of compacts having high density up to $90 \%$ of the theoretical value, for the powder extruded at room temperature (RT), with strain value of 3.17. Even better densities, 94 and $96 \%$ were achieved for extrusions at 700 and $800^{\circ} \mathrm{C}$, respectively, with lower strain values, 2 and 1.85, respectively. However, HE of powders at elevated temperatures led to grain growth, which resulted in deterioration of the magnetic properties.

Initial investigations showed that $\mathrm{HE}$ at room temperature also lowers the magnetic properties. Mechanism of

\footnotetext{
* corresponding author; e-mail:

tomasz.gizynski@inmat.pw.edu.pl
}

this phenomenon is unknown. The major, hard magnetic $\mathrm{Nd}_{2} \mathrm{Fe}_{14} \mathrm{~B}$ phase is brittle and does not undergo deformation. X-ray phase analysis did not show changes in the phase constitution after extrusion.

The aim of the current studies is determination of changes in the microstructure of hydrostatically extruded $\mathrm{Nd}-\mathrm{Fe}-\mathrm{B}$ powder, which are responsible for the deterioration of the magnetic properties.

\section{Experimental}

For the studies the MQU-F42 high coercivity powder, applicable for hot working, was used. The powder had composition $\mathrm{Nd}_{13.95} \mathrm{Fe}_{73.27} \mathrm{~B}_{5.49} \mathrm{Co}_{6.72} \mathrm{Ga}_{0.57}$ (at.\%) and was in a form of flakes, having thickness of $25 \mu \mathrm{m}$ and much greater other dimensions. The powder particles consisted of nanocrystalline grains of the $\mathrm{Nd}_{2} \mathrm{Fe}_{14} \mathrm{~B}$ phase, having mean diameter of $30 \mathrm{~nm}$. The theoretical density of the material was $7.5 \mathrm{~g} / \mathrm{cm}^{3}$.

The powder was initially densified in a copper capsule to about $60 \%$ of theoretical density. Subsequently the capsule was sealed under vacuum and welded with electron beam. The billet was hydrostatically extruded at room temperature, in the three-stage process, with application of various diameter reduction ratios, for each stage (Table). In the course of extrusion the copper billet was placed in a working chamber and surrounded by liquid pressured medium. Moving piston generated hydrostatic pressure. When the desired pressure was reached (it depends on the billet material and reduction ratio) the billet was extruded through the die. The final product was in a form of a rod with the $\mathrm{Nd}-\mathrm{Fe}-\mathrm{B}$ core.

The magnetic properties of the samples were examined using a Lake Shore vibrating sample magnetometer. 
TABLE

Selected parameters of hydrostatic extrusion and material properties after the process.

\begin{tabular}{c|c|c|c|c|c|c}
\hline \hline Process & $\begin{array}{c}\text { Diameter } \\
\text { reduction } \\
{\left[\mathrm{d}_{\mathrm{o}}^{2} / \mathrm{d}_{\mathrm{f}}^{2}\right]}\end{array}$ & $\begin{array}{c}\text { True } \\
\text { strain } \\
\varepsilon_{\mathrm{r}}[\%]\end{array}$ & $\begin{array}{c}\text { Density } \\
\rho \\
{\left[\mathrm{g} / \mathrm{cm}^{3}\right]}\end{array}$ & $\begin{array}{c}\text { Coercivity } \\
H_{\mathrm{c}}[\mathrm{kA} / \mathrm{m}]\end{array}$ & $\begin{array}{c}\text { Remanence } \\
B_{\mathrm{r}}[\mathrm{T}]\end{array}$ & $\begin{array}{c}\text { Lattice } \\
\text { deformation } \\
{[\%]}\end{array}$ \\
\hline Initial & - & - & 4.52 & 1690 & 0.750 & - \\
powder & - & 1.38 & 5.48 & 1476 & 0.544 & 0.0485 \\
Stage 1 & 2 & 0.89 & 6.84 & 1477 & 0.583 & 0.0974 \\
Stage 2 & 1.56 & 0.94 & 6.89 & 1366 & 0.577 & 0.0989 \\
Stage 3 & 1.6 & 0.69
\end{tabular}

Samples were characterized by XRD with $\mathrm{Cu} K_{\alpha}$ radiation. The crystalline lattice deformation of the $\mathrm{Nd}_{2} \mathrm{Fe}_{14} \mathrm{~B}$ crystallites was determined using the Williamson-Hall method [4]. The Mössbauer results were recorded using a POLON spectrometer, working in a transmission configuration with the constant acceleration of the Mössbauer source ${ }^{57} \mathrm{Co}$ in an $\mathrm{Rh}$ matrix, having the source activity of $50 \mathrm{mCi}$. The quantitative analysis was done using the WinNormos for Igor package.

\section{Results}

The properties were studied for initial material and after each stage of extrusion. It was found that the remanence of the material increased with growing density (Table). However, for greatest deformation (stage 3 ) the remanence decreased even for the high value of density. The coercivity decreased with increasing density.

Analysis of the microstructure showed that the powder particles, in the course of extrusion, experienced noticeable refinement. Due to the change of size and shape, the particles, are able to relocate in the course of extrusion. As a result of densification the overall area of the powder particles and the area of boundaries between them increase. Materials processed by $\mathrm{HE}$ are characterized also by a strong lattice deformation. These phenomena can negatively affect the magnetic properties. Their influence is very difficult to quantitatively assess, however, one can assume that they should not be the major reason for the decrease of the magnetic properties. The X-ray phase analysis, performed after hydrostatic extrusion, showed neither changes in the phase constitution nor peaks intensity and locations.

For the investigations with the use of the Mössbauer spectroscopy, in order to record a representative spectrum for the entire volume, the specimens were pulverised. Both materials, the initial powder and the extruded rod, after the three-stage process, were examined. In the analysis of the Mössbauer spectra for the initial powder the presence of the main, hard magnetic $\mathrm{Nd}_{2} \mathrm{Fe}_{14} \mathrm{~B}$ phase was identified. For the $\mathrm{Nd}_{2} \mathrm{Fe}_{14} \mathrm{~B}$ component, a presence of the four crystalographically and six magnetically non-equivalent positions of the Fe atom, in the elementary unit of this phase, were considered (Fig. 1).

In fitting the experimental spectrum, for the threestage extruded specimen, beside the six components,

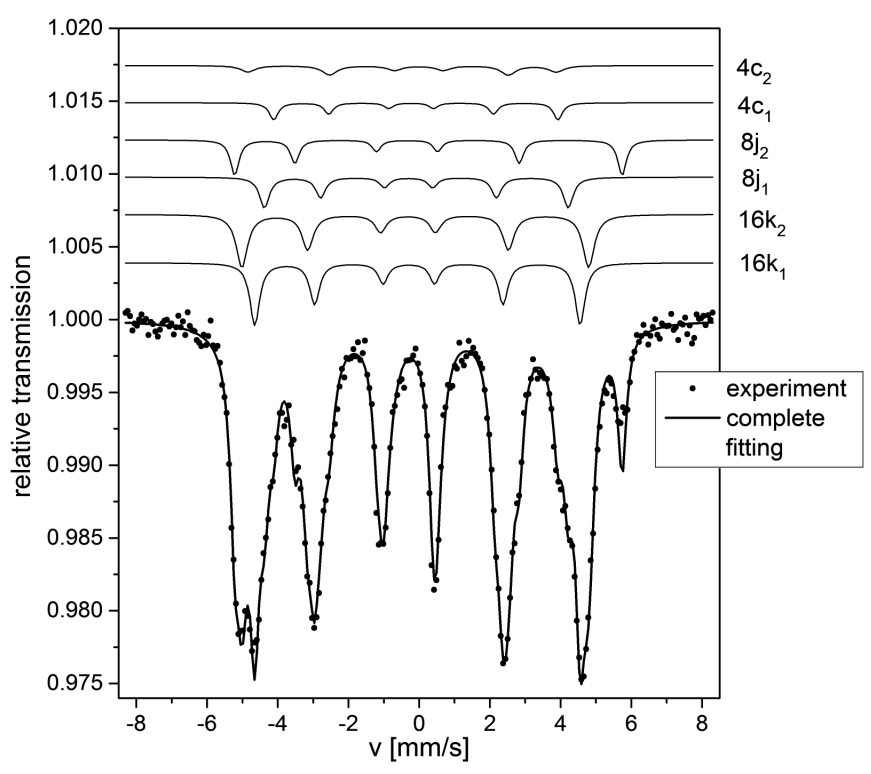

Fig. 1. Mössbauer spectra for the starting powder.

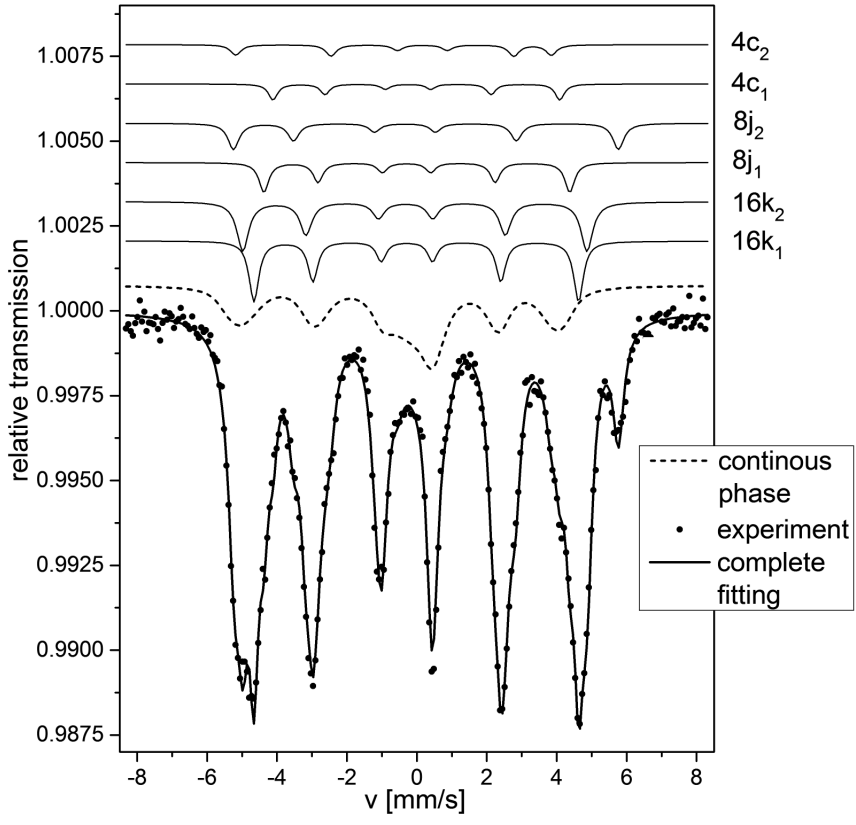

Fig. 2. Mössbauer spectra for the three-stage extruded specimen.

related to the hard magnetic $\mathrm{Nd}_{2} \mathrm{Fe}_{14} \mathrm{~B}$ phase, one continuous component, for which the distribution of the hyperfine fields was described, was taken into account. The presence of the continuous component can be related to either a presence of an amorphous or strongly disordered phase, which is formed during extrusion (Fig. 2). In Fig. 3 distribution of the hyperfine fields $P\left(B_{\mathrm{hf}}\right)$ for this phase is presented. In the plot one can distinguish two components of this distribution - low- and highfield, respectively. This evidences the existence of two various surroundings of the iron nuclei, in the disordered phase, having different local magnetic properties. In the extruded specimen, the values of the parameter such 
as $B_{\mathrm{hf}}$, for the hard magnetic phase attain higher values than for the initial powder. This can evidence the presence of internal stresses in the elementary unit cell of this phase, related to the characteristics of the hydrostatic extrusion process. On the basis of the analyses of areas of the sextet's components we found that the fractions of the hard magnetic and disordered phases in the material amount to $84 \%$ and $16 \%$, respectively.

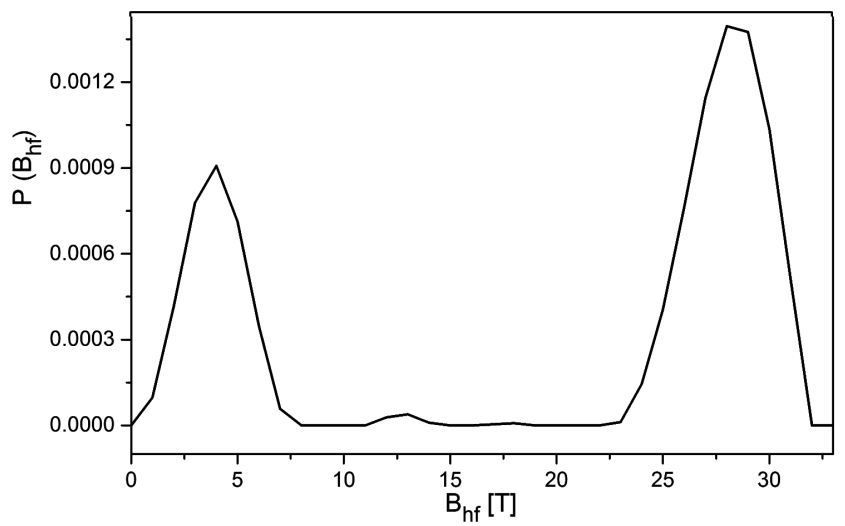

Fig. 3. Distribution of hyperfine fields for the threestage extruded specimens.

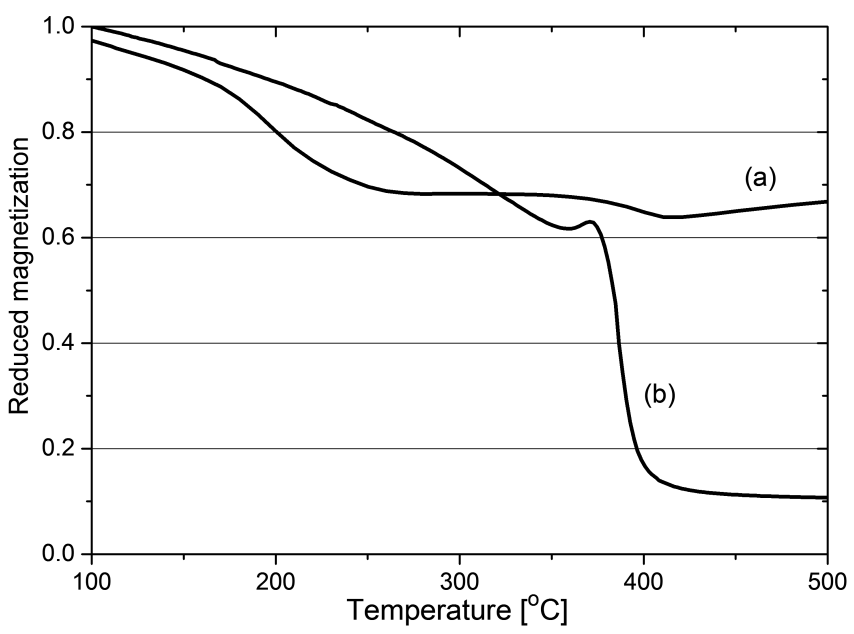

Fig. 4. Magnetization versus temperature for the three-stage extruded specimens $(a)$ and initial powder $(b)$.
In Fig. 4 magnetization versus temperature for the three-stage extruded specimens $(a)$ and initial powder $(b)$ are presented. Analysis of these dependences showed a presence of two, distinct deflection points for the extruded specimen (curve $a$ ). An abrupt drop of the magnetization, at a temperature of $390^{\circ} \mathrm{C}$ is characteristic of the Curie temperature for the $\mathrm{Nd}_{2}(\mathrm{Fe}, \mathrm{Co}){ }_{14} \mathrm{~B}$ phase. The other drop, observed around $200^{\circ} \mathrm{C}$ is apparently correlated with the existence of the strongly disordered phase. The parallel deterioration of the magnetic properties at this temperature is an evidence of the formation of the new ferromagnetic phase during extrusion.

\section{Conclusions}

The experimental data evidence that hydrostatic extrusions of the $\mathrm{Nd}-\mathrm{Fe}-\mathrm{B}$ powder leads to formation of a new, strongly disordered phase, probably an alternative of the $\mathrm{Nd}_{2} \mathrm{Fe}_{14} \mathrm{~B}$ phase. Appearance of this phase in the material apparently leads to deterioration of the magnetic properties of the extruded material.

\section{Acknowledgments}

The study was financed within the project co-funded by the European Union in Operational Programme Innovative Economy POIG 01.01.102.00/10 (ZAMAT). The powders were kindly provided by Magnequench.

\section{References}

[1] W. Pachla, M. Kulczyk, A. Świderska-Środa, M. Lewandowska, H. Garbacz, A. Mazur, K.J. Kurzydłowski, in: Proc. 9th Int. Conf. on Mat. Forming ESAFORM-2006, Glasgow (UK), Eds.: N. Juster, A. Rosochowski, Publishing House Akapit, Krakow, Poland 2006, p. 535.

[2] W. Pachla, A. Morawski, P. Kovac, I. Husek, A. Mazur, T. Lada, R. Diduszko, T. Melisek, V. Strbık, M. Kulczyk, Supercond. Sci. Technol. 19, 1 (2006).

[3] W. Kaszuwara, M. Kulczyk, M. Leonowicz, T. Gizynski, B. Michalski, IEEE Trans. Magn. 50, 1 (2014).

[4] D. Oleszak, A. Olszyna, Composites R4 11, 284 (2004). 